This is the final peer-reviewed accepted manuscript of:

Tian, V. Degli-Esposti, E. M. Vitucci and X. Yin

"Semi-Deterministic Radio Channel Modeling Based on Graph Theory and Ray-Tracing"

in IEEE Transactions on Antennas and Propagation, vol. 64, no. 6, pp. 24752486, June 2016

The final published version is available online at:

https://doi.org/10.1109/TAP.2016.2546950

Rights / License:

This article may be used for non-commercial purposes in accordance with Wiley Terms and Conditions for Use of Self-Archived Versions. The terms and conditions for the reuse of this version of the manuscript are specified in the publishing policy. For all terms of use and more information see the publisher's website.

This item was downloaded from IRIS Università di Bologna (https://cris.unibo.it/)

When citing, please refer to the published version. 


\title{
Semi-Deterministic Radio Channel Modelling Based on Graph Theory and Ray-Tracing
}

\author{
Li Tian, Vittorio Degli-Esposti, Enrico M. Vitucci, Xuefeng Yin
}

\begin{abstract}
Graph theory channel modelling is an efficient approach to simulate multipath radio propagation including the reverberation effect of electromagnetic waves. In this contribution, without modifying the modelling framework, we proposed a semi-deterministic channel modelling approach by associating the scatterers with realistic environment objects, and by calculating the coefficients of the propagation paths on the base of a physically sound and proven diffuse scattering theory. The diffuse multipath components are then combined with the specular components simulated by ray-tracing to obtain a complete channel representation. The proposed method is evaluated in two reference scenarios at 3.8 and $60 \mathrm{GHz}$ respectively by comparing the simulated channel characteristics with channel measurement data. Results show that the proposed method can accurately predict the channel characteristics in both the delay domain and the angular domain. The proposed approach is appropriate to model multipath propagation in confined indoor or dense-urban environment at millimeter-wave frequencies and above, where reverberation and rough-surface scattering can be important phenomena.
\end{abstract}

Index Terms-propagation channel modelling, diffuse scattering, millimeter wave, graph theory, ray-tracing

\section{INTRODUCTION}

$\mathbf{U}$ Rban multipath radio propagation can be studied by dividing the propagating field into two components, the specular component (SC) and the diffuse component (DC). From a propagation modelling point of view, the former consists of the direct wave and of the waves undergoing specular interactions such as reflections on surfaces and diffractions on edges. The latter consists of waves undergoing diffuse scattering due to interactions with small (compared to the wavelength) objects and with irregularities in the surface or in the volume of walls and major objects, and differently from the SC it's a distributed process, e.g. every surface element of a rough wall is a source of diffuse energy. At millimeter $(\mathrm{mm})$-wave frequencies, due to the very limited through-wall penetration and to the small wavelength, the DC is mainly generated by rough-surface scattering.

The DC has been found to have an important impact on the time-, space- and polarization-dispersion characteristics of the urban propagation channel [1]-[5]. The ratio of the DC vs. the $\mathrm{SC}$ has an important impact for example on the performance of

Manuscript received, $12^{\text {th }} 2014$

L. Tian and X. Yin are with the College of Electronics and Information Engineering, Tongji University, Shanghai, 201804, China (e-mails: \{09_tianli; yinxuefeng\}@tongji.edu.cn).

V. Degli-Esposti and E. M. Vitucci are with the Alma Mater StudiorumUniversità di Bologna, Dipartimento di Ingegneria dell'Energia Elettrica e dell'Informazione “Guglielmo Marconi” (DEI), IT-40136 Bologna, Italy (emails: \{v.degliesposti; enricomaria.vitucci\}@unibo.it). future beamforming transmission techniques at mm-waves [6]. The DC has been studied and modelled into existing channel models in various ways. In experimental studies, the term dense multipath component (DMC) has been used to identify the propagating field that cannot be captured when extracting major propagation paths from MIMO measurements using high-resolution algorithms. The DMC contains not only the DC but also the reverberating field due to waves undergoing a very high number of specular interactions, let us say greater than 3 or 4 . Although their field contribution is small compared to the direct wave (when present) and to the waves undergoing 1 or 2 reflections, the number of such waves can be much higher in confined indoor environments, where a multi-path background with chaotic characteristics is generated that is very "dense" and very "spread" in both time and space, therefore similar to the DC. Separation of the reverberation component from the DC properly is very difficult if not impossible by experimental means, but studies have shown that the residual DMC power that cannot be resolved by the measurement system can be estimated and modelled through the RiMAX algorithm [7].

During the last 15 years deterministic models such as raytracing (RT), have been extended to include diffuse scattering from building walls - and therefore the DC - with the "effective roughness" (ER) approach [2], [8] or the "multiple-facets approach" [9]. Ray models including diffuse components yielded more realistic simulation results than models accounting for the SC only when compared to measured data. Other studies proposed comprehensive approaches to model indoor propagation that accounts for the SC and DC at the same time [10].

In order to properly describe radio propagation in urban and indoor environment, it is necessary to model both the SC and the DC, including multiple-bounce reverberation effects for each one of them. While reverberation of the SC can be modelled quite efficiently through ray tracing techniques, at least in small indoor environments, the same is not true for the DC: since diffuse scattering is an intrinsically distributed phenomenon, computing multiple-bounce scattering for all the possible combinations of surface elements and small objects would require a huge computational effort. Recently, a new geometric-stochastic channel modelling approach based on graph theory has been proposed, which allows to simulate multiple-bounce scattering up to an arbitrary number of interactions in a very efficient way. In graph-theory channel models, propagating paths are represented as sequences of branches (edges) connecting nodes (vertexes, representing scatterers) [11]. A propagation graph can be intuitively set up according to the propagation environment, and the channel 
impulse response (CIR) can be calculated analytically with a limited computational cost, even taking into account an infinite number of bounces. Several publications have dealt with the GM approach [12]-[14] and results have been shown to be consistent with the WINNER II model [15]. The graph-theory channel models proposed so far however are not based on a rigorous description of the propagation mechanisms and are therefore not suitable for deterministic propagation modelling.

In this contribution, without modifying the modelling framework, we propose a new generation of semi-deterministic graph-theory models based on the proven ER diffuse scattering theory where the scatterer's distribution and the corresponding propagation coefficients are jointly defined so as to satisfy basic propagation theory laws, such as the power-distance decay law, power balance, transmission attenuation, etc. Therefore, the need for measurement-based parameter tuning should be reduced to minimum. Unlike in the original stochastic propagation graph modelling, the graph in our method is set up by the aid of digital maps: walls and buildings are discretized into scatterers whose spatial distributions over planes or volumes reflect the shape of the actual obstacle they represent. As an alternative, our approach is directly applicable when the environment database is obtained through laser scanning as a point-scatterer cloud, which is an increasingly popular and fast technique to derive accurate environment descriptions [16]. The SC, which cannot be easily modelled through the graph theory approach, is computed using RT and then combined with the DC. The latter, thanks to the infinite-bounce simulation capability of the graph-based approach, can model the reverberation effect. In highly diffuse propagation conditions where the DC is dominant, such as propagation at terahertz frequencies, the whole radio channel might be modelled using the modified graph-modelling approach without resorting to the RT integration [17]. The implementation procedure of the proposed method is illustrated using a single wall scenario at $3.8 \mathrm{GHz}$ and an indoor office scenario at $60 \mathrm{GHz}$ respectively. The two frequency bands represent good candidates for future generation wireless systems. Performance is evaluated by comparing the channel characteristics in both the delay and the angular domains with complete RT simulations and empirical results obtained from real channel measurements.

The organization of this paper is as follows: Section II introduces briefly the basic idea of propagation graph modelling. In Section III, the procedure of the proposed deterministic graph modelling and the corresponding scattering theory are elaborated. The experimental evaluations for the performance of the proposed method in two reference scenarios are presented in Sections IV and V respectively. Section VI briefly investigates the different characteristics between SC and DC with multiple bounces of reverberation. Finally, conclusive remarks are addressed in Section VII.

\section{FRAMEWORK OF PROPAGATION GRAPH MODELLING}

By adopting graph theory, the propagation graph modelling approach has first been applied to predicting CIRs in [11]. A so-called propagation graph can be generated by taking into account the geometry of the environment, the setup of the

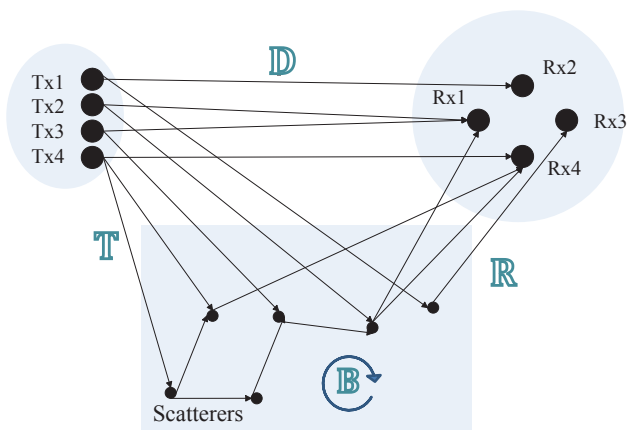

Figure 1. A configuration example of propagation graph.

transceivers, the mobility and visibility of the nodes, the scatterers' distribution, as well as the scatterers' electromagnetic (EM) properties.

For example, as shown in Figure 1, the position and visibility information of $m=4$ transmitters (Tx), $n=4$ receivers (Rx) and $s=5$ scatterers have been configured. The transfer function can be calculated in the frequency domain as [11]:

$$
\begin{aligned}
\mathbf{H}(f) & =\mathbf{D}(f)+\mathbf{T}(f)\left(1+\mathbf{B}(f)+\mathbf{B}(f)^{2}+\cdots\right) \mathbf{R}(f) \\
& =\mathbf{D}(f)+\mathbf{T}(f)(1-\mathbf{B}(f))^{-1} \mathbf{R}(f),
\end{aligned}
$$

where $f$ is the specific frequency band, $\mathbf{D}(f)$ with the size of $m \times n$ represents the line-of-sight (LOS) part of the transmission, and the rest items are the non-line-of-sight (NLoS) components induced by the reverberation of EM waves among scatterers. In (1), $\mathbf{T}(f)$ with the size of $m \times s, \mathbf{R}(f)$ with the size of $s \times n$ and $\mathbf{B}(f)$ with the size of $s \times s$ denote the transmission matrices with entries representing respectively the propagation coefficients of the links $e$ from individual transmitter to scatterers $\left(e \in \varepsilon_{t}\right)$, from scatterers to individual receiver $\left(e \in \varepsilon_{r}\right)$, and among scatterers $\left(e \in \varepsilon_{s}\right)$. $\varepsilon$ with different subscripts represent the sets of different propagation links determined by the pairs of the link ends. $\mathbf{B}(f)^{n}$ refers to the matrix for the $n$th bounce interactions between the scatterers. The transfer function of a propagation path that represents a link in Figure 1 can be calculated as:

$$
A_{e}(f)=g_{e}(f) \exp \left(-j 2 \pi \tau_{e} f+j \phi\right),
$$

where $A_{e}(f)$ depends on the elements of the matrices $\mathbf{D}(f), \mathbf{T}(f), \mathbf{B}(f)$ and $\mathbf{R}(f)$ according to different kinds of link ends, $\tau_{e}$ is the propagation delay or time of arrival, $\phi$ is a random phase rotation, which is uniformly distributed on the interval $[0,2 \pi)$, and $g_{e}(f)$ is the propagation coefficient depending on different kinds of links. The definition of $g_{e}(f)$ was originally proposed in [12]. In this contribution, we propose a modified deterministic definition, as will be discussed later in Section III-B.

The graph modelling approach is promising because of the following advantages. First, an environment can be easily modelled by either specifically or randomly generating a graph which contains the location information of the transceivers and scatterers. Second, CIRs with infinite bounces of propagation events are calculated in closed form based on the EM-wave reverberation theory. Third, the models can be generalized for 
Table I

COMPARISON OF THE COMPUTATION TIME (MINUTES) WITH DIFFERENT NUMBER OF BOUNCES OF DIFFUSE SCATTERING.

\begin{tabular}{|l||c|c|c|c|c|}
\hline Method Bounces & 0 & 1 & 2 & 3 & $\infty$ \\
\hline \hline Ray-tracing & 0.17 & 1.2 & 175 & $2.98 \times 10^{4}$ & N/A \\
\hline Graph modelling & N/A & 3.5 & 54 & 55 & 82 \\
\hline
\end{tabular}

specific frequency bands. Moreover, the complexity of graph modelling approach is limited. For example, the computation time of a single snapshot simulation with different number of bounces of diffuse scattering for an indoor office scenario with the area $5 \times 5 \times 3 \mathrm{~m}^{3}$ is compared in Table- I. The considered RT model is a full-3D Ray Tracing where the coherent components (specular reflection, diffraction) are computed using the classical Image Theory approach, in combination with Geometrical Optics (GO) and Uniform Theory of Diffraction (UTD). Diffuse scattering is also taken into account, and the scattering rays are computed using a ray-launching approach, i.e. launching rays from $\mathrm{Tx} / \mathrm{Rx}$ towards the walls/edges with a fixed angular discretization, both in azimuth and elevation. Further details about the RT model can be found in [18]. To be fair, in graph based simulation the environment is discretized into 1000 scatterers in order to have the same angular resolution $\left(1^{\circ}\right)$ as that used in RT simulations. It should be noticed that we did not count the time in building the visibility between the scatterers for the graph modelling, since it was settled down once we got the digitized map. From Table. I, it can be found that the computational cost of graph model is almost only related to the number of scatterers, doesn't grow rapidly with the number of bounces as with ray-based models. Thus, it will be very efficient to model the diffuse scattering with high number of bounces by using propagation graph modelling. RT is more practical when the scattering component is not dominant.

\section{PROPOSED DETERMINISTIC PROPAGATION GRAPH MODELLING}

The validity of propagation graph modelling approach has been investigated in several publications [11]-[14]. The results showed that the statistical characteristics of the propagation channel such as path loss and delay spread obtained by graph modelling are realistic. Those investigations are mainly based on randomly generated sets of graphs. For the same kind of environment, the statistical characteristics of the propagation channels simulated by graph modelling can be close to those obtained from measurements. However, since the definition of the propagation coefficients in [12] is not entirely based on the physical propagation mechanisms, the simulated power delay profiles (PDPs) may not be realistic enough for a site-specific approach unless extensive calibrations and parameterizations are conducted. Therefore, a deterministic propagation graph modelling is proposed to solve this problem [19], although the accuracy and physical soundness in the new approach come at the expense of generality.

\section{A. Deterministic graph modelling procedure}

The procedure for graph modelling in a given propagation environment consists of the following steps:

Step 1: Set the vertex information of the polygonal surfaces based on the digital map of the given scenario, and set the positions of the transceivers according to the scenario;

Step 2: Generate the locations of the scatterers, e.g. discretize every surface into multiple small tiles and assign a scatterer to represent each tile. Record the coordinates of the scatterers, and the normal directions of the surfaces are also recorded and assigned to the corresponding scatterers;

Step 3: Evaluate the visibility for every pair of two nodes (i.e. a Tx and a scatterer, a scatterer and a Rx, or two scatterers) according to their positions and the positions of the surfaces, which can be realized by the algorithm detecting whether the vector connecting the two nodes intersects the surfaces. A propagation graph is now set up;

Step 4: Calculate the propagation coefficient $g_{e}(f)$ for each available link and generate the matrices $\mathbf{D}(f), \mathbf{T}(f), \mathbf{R}(f)$ and $\mathbf{B}(f)$ according to (2) for specific frequencies;

Step 5: Embed the antenna radiation pattern for the matrices $\mathbf{T}(f)$ and $\mathbf{R}(f)$;

Step 6: Calculate the channel transfer function based on (1): the CIR is the inverse Fourier transform of the transfer function.

More detailed implementations can be found in Section IV and Section V for two specific scenarios. The framework of the proposed deterministic graph modelling is similar to that of the original graph modelling. However the calculation of the propagation coefficient $g_{e}$ is modified in order to satisfy power-balance and proper power-decay factors using the effective roughness scattering approach [20], as follows.

\section{B. Modifications on the propagation coefficients}

It should be noticed that diffuse scattering is the only interaction mechanism which is taken into account in the proposed deterministic graph modelling method. The reason for this choice is motivated by the following considerations. On the one hand, this model has been conceived for mm-wave applications, and it is known that rough-surface scattering can be very important at those frequencies, while diffraction and transmission can be neglected in most cases, compared to the lower frequency bands [4], [16]. Therefore, the proposed graph-theory-based method is suitable for modelling the propagation channel in mm-wave scenarios, also because the reverberation characteristics of the channel can be somehow modelled by means of multiple bounces of diffuse scattering, as will appear evident further on. On the other hand, graph modelling theory, being based on an iterative propagation process where each step only depends on the previous one, cannot easily model specular interactions that require to keep track of the whole geometrical history of the ray before computing the corresponding field contribution.

For more general applications where specular reflections are required, the proposed method can be combined with RT: the DC is generated by graph modelling and the SC is generated by RT. This approach is similar to the one 


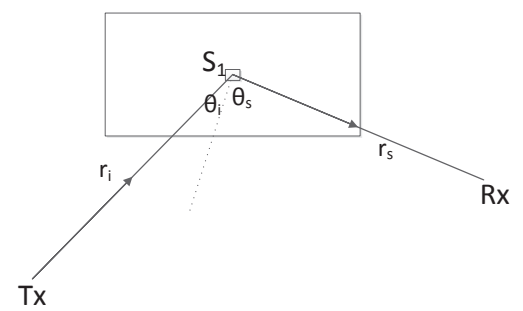

(a)

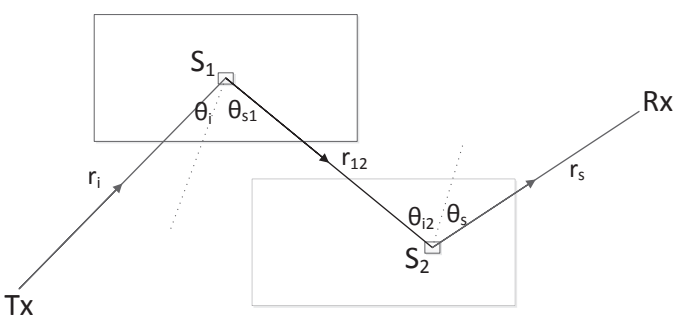

(b)

Figure 2. Illustrations of (a) single-bounce scattering and (b) double-bounce scattering respectively.

reported in [21], where RT simulations were combined with an analytical formula able to provide the power-angle and power-delay profiles of the diffuse components with high computational efficiency. However, the approach proposed in the present work is more general, because using the graph modelling we are able to deal with reverberating channels by taking into account an infinite number of successive diffuse interactions, while the analytical formula proposed in [21] was able to deal only with single-bounce scattering.

The following part will illustrate how to calculate the propagation coefficients $g_{e}$ based on the ER scattering model, with a Lambertian scattering pattern [8], [20].

First the single-bounce situation is considered, as shown in Figure 2(a). We intend to calculate the power transfer from the Tx to the Rx through a scattering path intercepted by a small tile at $S_{1}$ on the surface. If the transmit power is assumed to be $0 \mathrm{dBm}$ and both the Tx and Rx antennas are isotropic with $0 \mathrm{~dB}$ gain, according to the Friis equation [22], the field power at $S_{1}$ is

$$
E_{i}^{2}=\frac{1}{4 \pi r_{i}^{2}}
$$

where $r_{i}$ is the distance between the Tx and $S_{1}$, and $E_{i}$ is the module of the impinging field. Then back scattering from $S_{1}$ is modelled by using the Lambertian scattering pattern, and the amplitude of the scattering wave $E_{s}$ can be calculated as

$$
E_{s}=E_{s 0} \sqrt{\cos \left(\theta_{s}\right)},
$$

where $E_{s 0}$ is the maximal amplitude of the scattering wave towards the normal direction of the surface, and $\theta_{s}$ is the angle between the scattering wave and the normal direction of the surface. According to the scattering power balance, we have

$$
\begin{aligned}
S^{2} E_{i}^{2} d S \cos \left(\theta_{i}\right) & =\int_{0}^{\frac{\pi}{2}} \int_{0}^{2 \pi} E_{s}^{2} r_{s}^{2} \sin \left(\theta_{s}\right) \mathrm{d} \phi \mathrm{d} \theta_{s} \\
& =\pi E_{s 0}^{2} r_{s}^{2},
\end{aligned}
$$

where $S$ is the constant representing the scattering loss, $\mathrm{d} S$ denotes the area of the small tile at $S_{1}$, and $\theta_{i}$ is the angle between the incident wave and the normal direction of the surface. It means that the total scattered power at a distance $r_{s}$ from the small tile should be identical to the power intercepted by the small tile multiplied by $S^{2}$. Therefore, the power density at Rx can be derived as

$$
E_{s}^{2}=E_{s 0}^{2} \cos \left(\theta_{s}\right)=\frac{\mathrm{d} S \cos \left(\theta_{i}\right)}{4 \pi r_{i}^{2}} \cdot \frac{S^{2} \cos \left(\theta_{s}\right)}{\pi r_{s}^{2}} .
$$

Finally, the received power at the location $r_{s}$ away from $S_{1}$ following the direction $\theta_{s}$ can be calculated as

$$
P_{r}=E_{s}^{2} \cdot A_{\text {eff }}=\frac{\mathrm{d} S \cos \left(\theta_{i}\right)}{4 \pi r_{i}^{2}} \cdot \frac{S^{2} \cos \left(\theta_{s}\right)}{\pi r_{s}^{2}} \cdot \frac{\lambda^{2}}{4 \pi},
$$

where $\lambda$ is the wavelength and $A_{\text {eff }}=\frac{\lambda^{2}}{4 \pi}$ is the effective area for the isotropic antenna [23, p. 19].

Through factorization of the power transfer $P_{r} / P_{t}$ (the transmit power $P_{t}$ is assumed to be $0 \mathrm{dBm}$ as mentioned) into two parts, i.e. the incident part and the scattering part, the propagation coefficients for the links in $\varepsilon_{t}$ and $\varepsilon_{r}$ are

$$
g_{e}^{2}= \begin{cases}\frac{\mathrm{d} S \cdot \cos \left(\theta_{i}\right)}{4 \pi r_{i}^{2}} & e \in \varepsilon_{t} \\ \frac{S^{2} \cos \left(\theta_{s}\right)}{\pi r_{s}^{2}} \cdot \frac{\lambda^{2}}{4 \pi} & e \in \varepsilon_{r}\end{cases}
$$

Furthermore, the modified propagation coefficient for the links in $\varepsilon_{s}$ can be derived from the double-bounce situation, as shown in Figure 2(b). According to the previous derivations, the power density at $S_{2}$ is

$$
E_{s 2}^{2}=\frac{\mathrm{d} S \cos \left(\theta_{i}\right)}{4 \pi r_{i}^{2}} \cdot \frac{S^{2} \cos \left(\theta_{s 1}\right)}{\pi r_{12}^{2}},
$$

then the Lambertian scattering model is applied to the small tile at $S_{2}$ again, and the scattering power balance at $S_{2}$ can be described as

$$
S^{2} E_{s 2}^{2} \mathrm{~d} S \cos \left(\theta_{i 2}\right)=\pi E_{s 0} r_{s}^{2} .
$$

Therefore, the received power at $\mathrm{Rx}$ is calculated as

$$
\begin{aligned}
P_{r} & =E_{s}^{2} \cdot A_{\text {eff }}=E_{s 0}^{2} \cos \left(\theta_{s}\right) \cdot A_{\text {eff }} \\
& =\frac{\mathrm{d} S \cos \left(\theta_{i}\right)}{4 \pi r_{i}^{2}} \cdot \frac{S^{2} \cos \left(\theta_{s 1}\right)}{\pi r_{12}^{2}} \cdot \frac{S^{2} \mathrm{~d} S \cos \left(\theta_{i 2}\right) \cos \left(\theta_{s}\right)}{\pi r_{s}^{2}} \cdot \frac{\lambda^{2}}{4 \pi},
\end{aligned}
$$

where $\theta_{s 1}$ and $\theta_{i 2}$ are the scattering angle for $S_{1}$ and the incident angle for $S_{2}$ respectively, and $r_{12}$ is the distance between $S_{1}$ and $S_{2}$. Through factorization of the power transfer function, the modified propagation coefficients for all kinds of links are calculated as

$$
g_{e}^{2}= \begin{cases}\frac{\left(\frac{\lambda}{4 \pi r_{d}}\right)^{2},}{\frac{\mathrm{d} S \cdot \cos \left(\theta_{i}\right)}{4 \pi r_{i}^{2}},} & e \in \varepsilon_{d} \\ \frac{S^{2} \cos \left(\theta_{s}\right)}{\pi r_{s}^{2}} \cdot \frac{\lambda^{2}}{4 \pi}, & e \in \varepsilon_{t} \\ \frac{S^{2} \mathrm{~d} S \cos \left(\theta_{i 2}\right) \cos \left(\theta_{s 1}\right)}{\pi r_{12}^{2}}, & e \in \varepsilon_{s}\end{cases}
$$




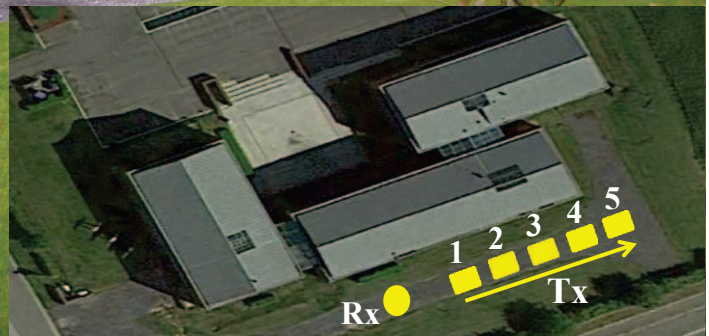

Figure 3. Measurement information of the isolated office scenario.

Table II

CHANNEL SOUNDING CONFIGURATION PARAMETERS.

\begin{tabular}{c|c}
\hline Frequency & $3.8 \mathrm{GHz}$ \\
Bandwidth & $200 \mathrm{MHz}$ \\
Transmit Power & $23 \mathrm{dBm}$ \\
Code Length & $5.11 \mu \mathrm{s}$ \\
Antenna Array & 4 elements, dual-polarized \\
Antenna Height & $1.5 \mathrm{~m}$ \\
\hline
\end{tabular}

where the coefficient in $\varepsilon_{d}$ (i.e. LoS link) is defined based on the free-space Friis equation.

\section{Performance evaluation through a single WALL SCENARIO}

The proposed deterministic graph modelling is applied to simulate the CIR for an isolated office building scenario [19]. Results are validated against the measurements data that are collected in such an environment and adopted to evaluate the validation of the proposed approach.

\section{A. Measurement setup}

The picture of the considered environment and the top view of the scenario are shown in Figure 3. The measurement took place in Louvain-la-Neuve (Belgium), in front of an isolated office building, characterized by a large glass wall sustained by a metallic frame [24]. Measurements were performed with the UCL-ULB Elektrobit MIMO Channel Sounder, which is based on a pseudo-random noise code technique. Its setup parameters are shown in Table II. Both the Tx and Rx antennas are linear horizontal arrays of 4 dual-polarized $(+45 /-45)$ patches. Both the arrays have been pointed towards the wall, i.e. with the aperture plane parallel to the wall plane. Each element has a gain of about $6 \mathrm{dBi}$, with a half-power beamwidth of about $95^{\circ}$. Measurements have been carried out in a static configuration, i.e. the Tx unit has been moved in 5 different positions as marked in Figure 3, and then a single snapshot has been recorded for each position. Linear arrays of 4 dualpolarized elements (slanted pol, $+/-45^{\circ}$ ) have been used in the measurements, so for each position we averaged all the 64 measured impulse responses to get the Power-Delay Profile.

\section{B. Environment representation for graph-based modelling}

Generated with the aid of the digital map of the measurement scenario, the positions of the Txs (red stars), the Rx (black star) and the scatterers (blue circles) are shown in

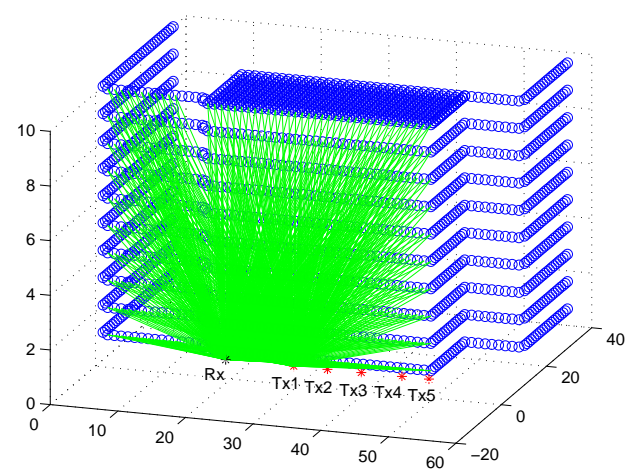

Figure 4. Setup of the propagation graph according to the site-specific measurement scenario.

Figure 4. The digital map was built by measuring the real size of each object and wall surface. The scatterers are generated by discretizing the existing walls in the environment. Each scatterer represents a small piece of tile with the area $\mathrm{d} S=1$ $\mathrm{m}^{2}$, and the scattering coefficient is defined as $S=0.6$ according to the evaluations in [24]. The generated scattering paths between Tx1 and Rx, for the given tile area are shown as green lines in Figure 4. Once the propagation graph has been set up, the formulas (1), (2) and (12) are used to calculate the CIRs.

\section{Performance comparison}

The comparison of the PDPs between different kinds of methods for Tx1 and Tx3 are shown in Figure 5 (a) and (b) respectively, where "Graph only scattering" means the proposed deterministic graph modelling approach based on the Lambertian scattering model, "Graph original" represents the original graph modelling approach based on the formulas proposed in [12] with the same scattering coefficient $S=0.6$, "RT, Lambertian" denotes the full-3D RT approach where the ER scattering model has been embedded in RT simulations using a Lambertian pattern [20], and "RT, only scattering" is also based on RT simulations, but taking into account only singlebounce ER scattering without reflection and diffraction. Since there is only one wall generating outward back-scattering in this scenario representation, no multi-bounce propagation is present here. The two curves showing the behavior of DC simulated respectively by graph modelling and RT are almost coincident, as it should be.

It can be observed from the comparison results that:

1) compared with the original graph modelling, the proposed method generates the PDPs with more realistic exponential decaying slopes. The $10-20 \mathrm{~dB}$ power overestimation in the original graph-modeling curve tail is due to the fact that the formulas used in [12] to calculate the propagation coefficients do not take into account power balance considerations nor parameters tuning vs. measurements.

2) the reflected peak is missing in the PDPs obtained with the proposed method as specular reflection is not considered. However, the later components i.e. diffuse tails are almost the 


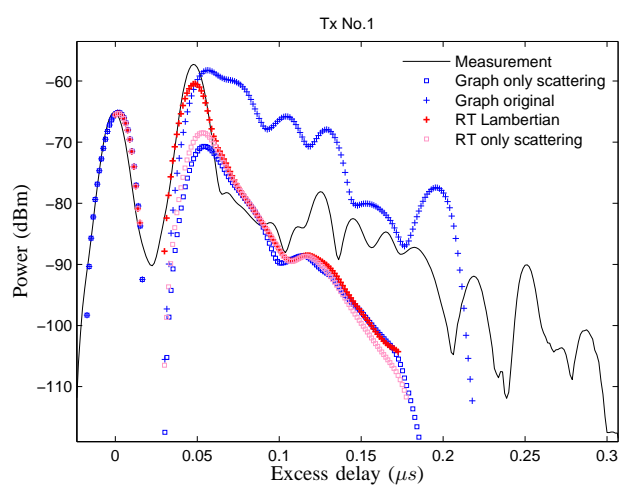

(a)

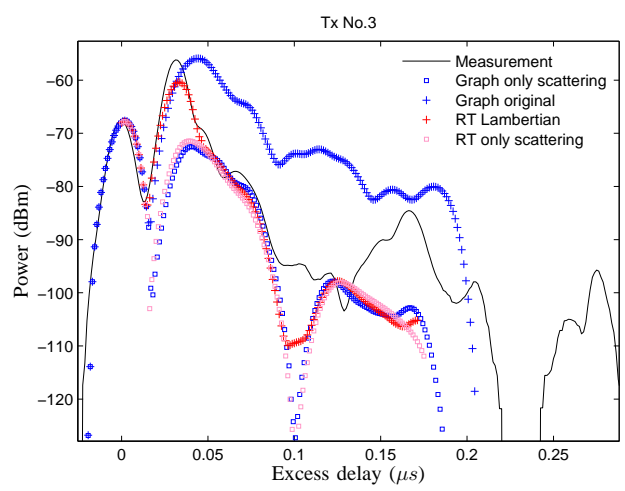

(b)

Figure 5. Comparison of the power delay profiles for (a) Tx1 and (b) Tx3 respectively.

same for both the RT method and our proposed method which implemented the same Lambertian model;

3) from the measured PDPs, specular reflection is very strong in this case, since the measurement campaign has been carried out at Super High Frequency (SHF). As mentioned in Section III-B, this mismatch can be solved by combining the diffuse scattering components simulated by graph modelling approach and the specular components simulated by RT method;

4 ) it is worth noticing that the simulated power of highdelay components is lower than the one of the measurements. We postulate that this is due to the fact that the considered environment is not actually a single wall but an office building with internal objects and furniture. There are some contributions in the tail of PDP due to rays which penetrate into the building and then are backscattered towards the receiver, after reflection/scattering on the internal structure of the building. Therefore, all these propagation mechanisms involving the internal building structures should be taken into account, to achieve a better match with the measurements.

\section{Including internal scatterers}

In order to account for propagation mechanisms taking place inside the building, some additional "volume scatterers" which represent the internal structures should be included. Since the exact internal structures are not available, the additional volume scatterers are distributed according to a proper volume discretization. In this case, since this additional scatterers do not belong to the walls, an isotropic scattering pattern is more suitable to describe their behaviour. The power contribution of each scatterer can be expressed as in [25]:

$$
P_{r}=\frac{P_{t} G_{t}}{4 \pi r_{i}^{2}} \sigma \frac{1}{4 \pi r_{s}^{2}} A_{\mathrm{eff}},
$$

where $\sigma$ represents the cross-section area of the scatterer. In this case, similar to (12), the propagation coefficients of the additional volume scatterers used for graph modelling are calculated as

$$
g_{e}^{2}= \begin{cases}\left(\frac{\lambda}{4 \pi r_{d}}\right)^{2}, & e \in \varepsilon_{d} \\ \frac{1}{4 \pi r_{i}^{2}}, & e \in \varepsilon_{t} \\ \frac{\sigma}{4 \pi r_{s}^{2}} \cdot \frac{\lambda^{2}}{4 \pi}, & e \in \varepsilon_{r} \\ \frac{\sigma}{\pi r_{12}^{2}}, & e \in \varepsilon_{s}\end{cases}
$$

Here, 100 internal scatterers are generated inside the building. $\sigma$ is set to be 5 to be compliant with power-balance consideration according to the volume of the building. In other words, assuming the internal scatterers as cubes of face area $\sigma$ and edge $\sqrt{\sigma}$, the overall volume occupied by $N$ scatteres, i.e. $N \sigma \sqrt{\sigma}$ should be lower than, or equal to, the volume of the building.

In order to take into account the transmission through the building walls, during the evaluation of the visibility between each node pair we also need to calculate the total number of wall crossings $\left(n_{e}\right)$. An empirical attenuation of $10 \cdot n_{e}$ $\mathrm{dB}$ is then added to the corresponding link $e$ by assuming the wave vertically impinging at the concrete/coated glass with the average thickness of $0.2 \mathrm{~m}$ at $3.8 \mathrm{GHz}$. The penetration loss can be also calculated in a deterministic way, with slightly more complexity. Moreover, although in theory the graph modelling approach is able to consider an infinite number of scattering bounces, in practice the power of the multiple-bounce contributions will attenuate drastically when the number of bounces and wall crossings increases. Therefore, the optimal number of diffuse scattering bounces is worth investigation. Figure 6 depicts the updated results by combining the SC generated by RT and DC with multiple bounces generated by the proposed graph modelling. It can be observed that after including the contributions of the internal scatterers, the tails of the PDPs get much closer to the measured results. By comparing the PDPs with the measured one, three bounces of diffuse scattering is identified to be enough to describe the reverberation phenomenon in this scenario according to Figure 6. In other words even if the internal structure of the building is unknown, the contribution of propagation inside the building to the CIR can be simulated through multiplebounce DC computed by graph modelling by assuming some internal scatterers. Although there are still some mismatched components beyond $0.25 \mu \mathrm{s}$, considering the large propagation distance, it can be postulated that those component are not due to the propagation from the office building, but probably from 


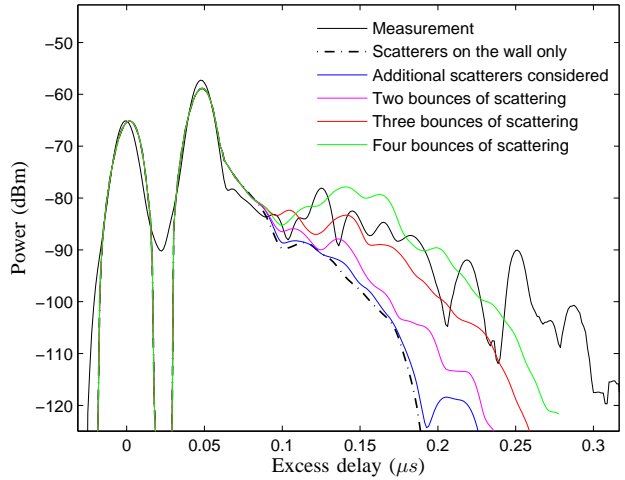

Figure 6. Comparison of the power delay profiles for Tx1, with SC and additional scatterers considered.

some other objects such as the trees and the distant buildings around.

Two metrics, i.e. total received power and delay spread are employed to evaluate the modelling accuracy, and $30 \mathrm{~dB}$ below the maximum power was selected as the threshold for the calculation of the metrics. the total power is calculated as

$$
P=\int_{\tau} P(\tau) d \tau
$$

and the delay spread as

$$
\sigma_{\tau}=\sqrt{\frac{\int_{\tau} P(\tau) \cdot(\tau-\bar{\tau})^{2} d \tau}{\int_{\tau} P(\tau) d \tau}},
$$

where the mean delay $\bar{\tau}=\frac{\int_{\tau} P(\tau) \cdot \tau d \tau}{P}$. The comparisons of the received power and delay spread between different approaches for all the 5 Tx positions are depicted in Figure 7 and 8 respectively. Here, "RT specular" denotes the full-3D RT approach which only considers the specular paths, while an extra single-bounce ER scattering model is embedded in "RT Lambertian". "Graph single bounce" and "Graph 3 bounces" represent the graph modelling approaches which take into account the SC combined with single bounce and three bounces of DC respectively. "Graph original" represents the original graph modelling approach based on the formulas proposed in [12]. It can be observed from the two figures that: $(i)$ the total channel power usually has a $3 \mathrm{~dB}$ underestimation if diffuse scattering is not considered, which means almost $50 \%$ of the power might be attributed to the DC in this scenario. The LoS and specular reflection path is indeed strong, but the number of scattering paths is even huge, i.e. 2000+. Therefore, although a single scattering path contributes only a little (i.e. $30 \mathrm{~dB}$ lower than the reflection), the total scattering power is comparable to the reflected power. However some architectural elements (indentations, metal rods, rain gutters etc) are missing in RT simulation: if these could be added, probably the residual power percentage attributable to the DC would be lower. (ii) the introduction of the additional volume scatterers and multiple bounces of DC have very little influence on the received total power, but it can effectively improve the delay spread since the slopes and tails (paths with

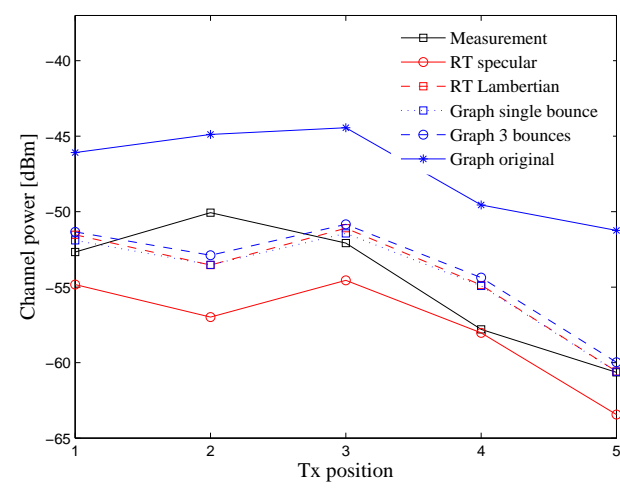

Figure 7. Received power: comparison between measurement and simulation with different approaches.

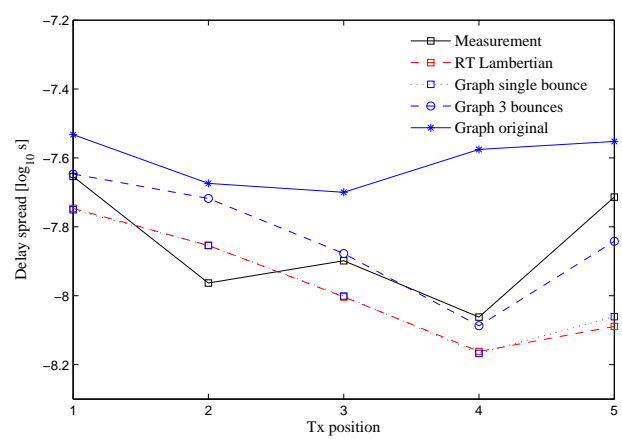

Figure 8. RMS delay spread: comparison between measurement and simulation with different approaches.

large delays) of the simulated PDPs are more realistic. (iii) the original graph modelling approach is not capable enough to simulate the propagation channel with reasonable total power and delay spread directly from a given digital map. Although results might be improved by tuning the parameters (number of scatterers, distribution of scatterers, scattering coefficient, etc.) to fit the measured results, this cannot be done without measurement data. Our proposed deterministic graph model is more applicable in this respect as the definition of the propagation coefficients has a physical meaning, and thus it can be directly applied to various scenarios.

\section{Performance EVAluation in AN INDOOR OFFICE SCENARIO}

In this section, the proposed modelling approach is applied to simulate the CIR in an indoor office scenario at mm-wave frequency. Ray-tracing prediction and measurements are used for comparison.

\section{A. Description of the environment and measurement setup}

The measurement is carried out in a small office located in the basement of the Technical University of Ilmenau, Germany, as described in [26]. The M-sequence ultra wide-band channel sounder used for the measurements consists of one Tx and two 


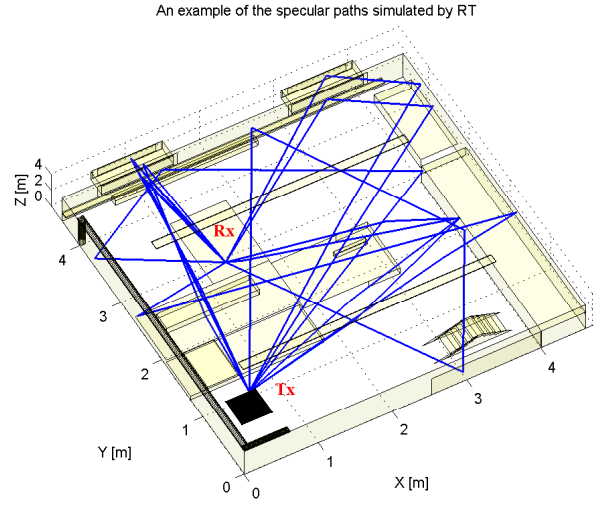

Figure 9. An example of specular paths simulated by ray-tracing.

Rx channels, Rx1 and Rx2. Only Rx1 with NLoS condition is considered in the investigation of diffuse scattering. The carrier frequency is $60 \mathrm{GHz}$ and the bandwidth is $3 \mathrm{GHz}$. The Tx equipped with a high gain lens antenna $\left(5^{\circ}\right.$ beamwidth) is located high in a corner of the room emulating an access point, and the RX is put on a desk. The deployment is an obstructed line-of-sight (OLOS) scenario where the direct link between TX and RX is obstructed by 2 absorbing panels. During the measurement, the TX swept a quarter of a sphere, with an angular step of $2^{\circ}$ from $0^{\circ}$ to $90^{\circ}$ in azimuth and $-60^{\circ}$ to $30^{\circ}$ in elevation, while the receiver with omni-directional antennas was fixed to perform static measurements.

\section{B. Implementation procedure of the proposed modelling ap- proach}

A digitized map of the office environment was built for the simulation. The implementation procedure for this scenario is similar with the previous one, but still there are some differences due to the more complex environment.

(1) Based on the digitized map, a 3D ray-tracing tool [2] is adopted to calculate the SC (i.e. reflections and diffractions with multiple bounces), as shown in Figure 9.

(2) The setup of the propagation graph, i.e. the positions of the Tx (red star), the Rx (black star) and the scatterers (yellow and blue circles) are shown in Figure 10. It should be noticed that only a reduced set of the scatterers is shown here, for the sake of clarity. Differently from the previous single wall scenario, the surfaces here are classified into two groups. Scattering tiles of area $\mathrm{d} S_{1}=0.01 \mathrm{~m}^{2}$ and scattering coefficient $S_{1}=0.2$ are assumed for the smooth surfaces (yellow circles) such as tables, display screens and metal boxes, while $\mathrm{d} S_{2}=0.04 \mathrm{~m}^{2}$ and $S_{2}=0.6$ is assumed for the rough surfaces (blue circles) such as walls and bookshelves filled with books, respectively. The calibration of $S_{2}$ parameter has been investigated and calibrated in [6]. The smoother the surface is, the less scattering power should be. Therefore, $S_{1}$ is empirically chosen to be smaller than $S_{2}$. The sizes of the tiles are set to be much smaller than those in the previous single wall scenario, in order to have enough angular resolution.

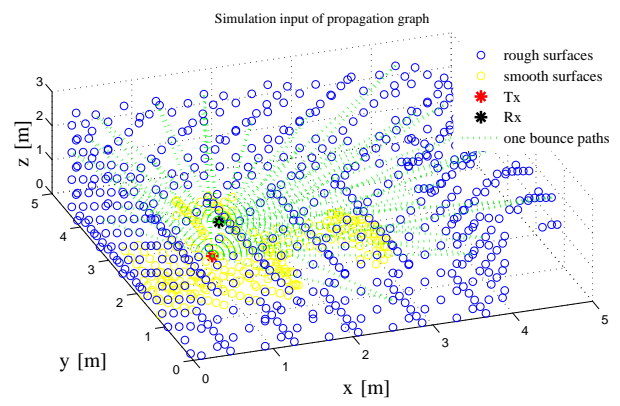

Figure 10. Implementation of the propagation graph in the office scenario.

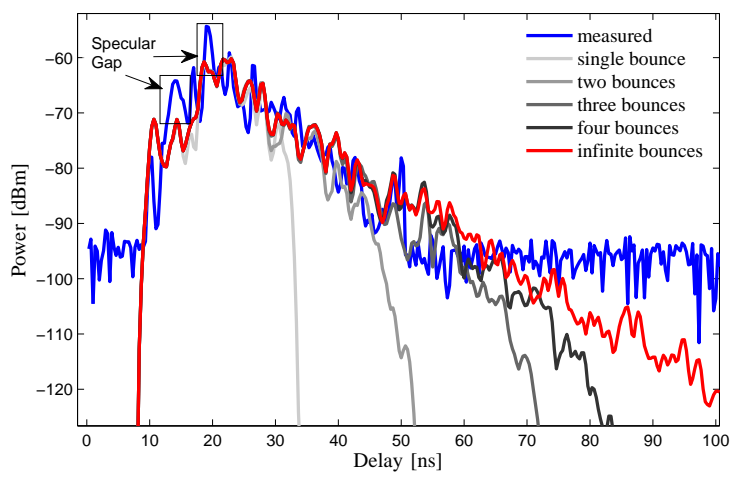

Figure 11. Comparison of the power delay profiles between the measurement and graph modelling (DC only).

(3) During the evaluation of visibility, when there is a direct link between a node pair, the propagation coefficient $g_{e}(f)$ is computed according to (12). Otherwise, when the link is obstucted by obstacles, an additional transmission loss is added according to the EM properties and thicknesses of the materials, similarly to what done in the single building case. The actual one-bounce propagation paths (i.e. when the scatterers are both visible to $\mathrm{Tx}$ and $\mathrm{Rx}$ ) are also shown in Figure 10;

The remaining steps, i.e. the calculation of the matrices $\mathbf{D}(f), \mathbf{T}(f), \mathbf{R}(f), \mathbf{B}(f)$ and the realizations of CIR are the same as shown in Section III.

\section{Performance evaluation in the delay domain}

The comparison of the PDPs between the measurement and graph modelling (DC only) is shown in Figure 11, where $n$ bounces $(n=1,2, . ., \infty)$ denote different simulated PDPs by using the graph modelling with the reverberation up to $n$ bounces. The dynamic range for the measurement is about $40 \mathrm{~dB}$ and the simulated PDPs are shown without noise in order to investigate the differences between different orders of reverberation.

It can be observed that:

1) with limited calibration of the parameters, the graph modelling is able to generate the PDPs with realistic power decaying slopes;

2 ) it can be deduced that at least 3 bounces of diffuse scattering are sufficient to describe the reverberation phenomenon 


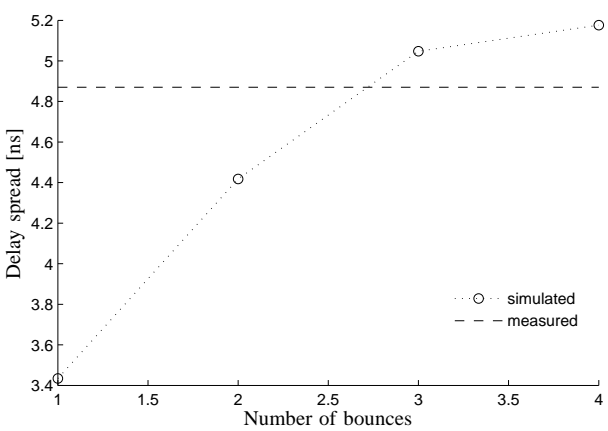

Figure 12. Delay spread comparison between measurement and graph modelling (DC only).

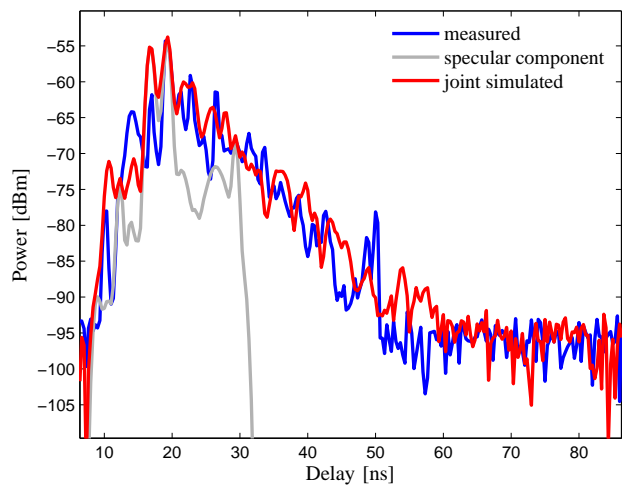

Figure 13. Power delay profiles comparison between measurement and joint simulation.

in this scenario. This is also supported by the comparison of the delay spread, as shown in Figure 12. Unfortunately, we could not compare the tail of PDPs beyond a maximum delay of $60 \mathrm{~ns}$ due to the dynamic range limit in the measurement. Nevertheless, the power level of those components is close to noise level and therefore not very significant;

3) the initial SC peaks (marked with "specular gap") are obviously missing in the PDPs obtained by graph modelling. However, this gap (about $6 \mathrm{~dB}$ ) is smaller comparing to the previous single wall case which was conducted at SHF (more than $10 \mathrm{~dB}$ ). It proves that the DC plays a more important role at the higher frequencies. As mentioned, this mismatch will be solved by combining the DC simulated by graph modelling and the SC simulated by RT.

Figure 13 shows the comparison result between the joint simulated PDP and the measured one. In this simulation, 3 bounces of diffuse scattering have been set, and these diffuse components have been combined with the specular components simulated with Ray Tracing. In the RT simulation up to 3 reflections, 2 diffractions and 10 transmissions have been considered. The strongest specular propagation paths are depicted in Figure 9. Since the impulse responses generated by RT have an infinite bandwidth, the corresponding PDPs have to be filtered according to the measurement set-up bandwidth. Besides, the same noise floor is added to the simulated PDP to obtain a fair comparison. From Figure 13, it can be found

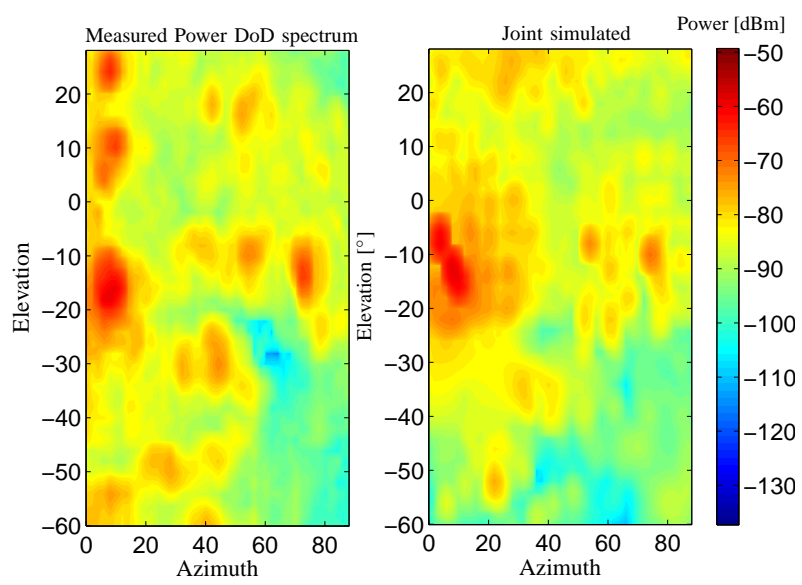

Figure 14. Comparison of the power angular spectra between the measurement and joint simulation, colors represent the powers.

that the combined PDP is consistent with the measured one in terms of main peaks, overall power level and decaying slope. It should be noticed that there is still a specular gap at around 15ns. Considering that the delay is even less than the first reflection at 20ns, we think it was possibly caused by the diffraction/transmission from the non-ideal absorber which is assumed to be an ideal one during the simulation.

\section{Performance evaluation in the angular domain}

In order to study the channel characteristics in the angular domain with the graph modelling approach, the definition in (1) should be slightly modified in the following way:

$$
\mathbf{H}\left(f, \Omega_{\mathrm{DoD}}\right)=\left\{\mathbf{D}(f), \mathbf{T}(f) * *\left[(1-\mathbf{B}(f))^{-1} \mathbf{R}(f)\right]^{T}\right\},
$$

and

$$
\mathbf{H}\left(f, \Omega_{\text {DoA }}\right)=\left\{\mathbf{D}(f),\left[\mathbf{T}(f)(1-\mathbf{B}(f))^{-1}\right] * \mathbf{R}(f)^{T}\right\} .
$$

The entries of the former vector denote the power transmitted to each scatterer and the $\mathrm{Rx}$, where $\Omega_{\mathrm{DoD}}$ consists of the direction of departures (DoDs) between the Tx and the other vertices. And the entries of the latter vector denote the power received from the Tx and each scatterer, where $\Omega_{\mathrm{DoA}}$ consists of the direction of arrivals (DoAs) between the Rx and the other vertices. $(\cdot)^{-1}$ denotes the matrix inverse, $[\cdot]^{\mathrm{T}}$ means the vector transpose and .* is the dot product of two vectors. By calculating the DoDs and DoAs according to the locations of the vertices in the propagation graph, the angular profiles can be derived as

$$
P\left(\Omega_{\mathrm{DoD}}\right)=\int\left|\mathbf{H}\left(f, \Omega_{\mathrm{DoD}}\right)\right|^{2} \mathrm{~d} f
$$

and

$$
P\left(\Omega_{D o A}\right)=\int\left|\mathbf{H}\left(f, \Omega_{\text {DoA }}\right)\right|^{2} \mathrm{~d} f,
$$

respectively. A similar procedure is also performed to derive the angular profile of the specular components generated by RT. 
Figure 14 shows the comparison between the measured and simulated power DoD spectra. It can be observed that the most dominant SCs are captured by RT simulation, and also the DMC (background) is simulated accurately. There are still some mismatches, for example, the two components with the azimuth angles around $10^{\circ}$ and the elevation angles equals to $10^{\circ}$ and $20^{\circ}$ respectively are not predicted in the simulated PAS. Based on the power level and our visual inspection, these are corresponding to either single or doublebounce reflection caused by the lamp on the ceiling and the window frame on the back wall. In the digitized map, the lamp and the window frame are simply modelled as cuboids. The metal structure of the light-reflectors inside the lamp which probably cause the reflections haven't been accurately described, hence the mismatch. Nevertheless, we think this accuracy is acceptable considering the complexity of the NLoS propagation environment. The details of the environment such as those small objects around the Rx and the aforementioned metal materials inside the lamp are not easy to be modelled and included in the digitized map. The other reason could be that in order to measure the power DoD spectrum, the TX swept in the sphere with an angular step of $2^{\circ}$, while the beam-width of the lens antenna is $5^{\circ}$. There would be some overlaps of the radiation patterns when measuring the impulse responses of different directions, but this effect is not taken into account in our model for the sake of simplicity. The accuracy of our model can be enhanced at the condition of a more refined digitized map.

Finally, as shown in Table-III, several large-scale channel characteristics are compared between the measurement and simulation. $30 \mathrm{~dB}$ below the maximum power was selected as the threshold for the calculation of the metrics, so as to remove the impact of noise. Besides, the values suggested by WINNER II model (A1 Office Scenario) are also presented as a reference [15]. Since only static measurement data are available, time-variant or statistical parameters such as Doppler frequency shift, Rician K-factor and cross-correlations are not considered here. It can be observed that, the simulated channel characteristics considering both DC and SC are in accordance with the measured results in terms of total channel power, delay spread, azimuth spread and elevation spread, while the simulation which only takes SC into account would underestimate the large-scale characteristics due to the lack of ubiquitous diffuse scattering. Moreover, These values (especially delay spread) are typically less to those of WINNER model due to the fact that: 1) during the measurement, the Tx only swept a quarter of the whole sphere, so there are many propagation paths not observed in the measured data; 2) the WINNER II model was constructed for the frequency band of $2-5 \mathrm{GHz}$, while at millimeter-wave frequency bands, because of the much higher free-space propagation loss, penetration loss and diffraction loss, power decaying rate along the propagation delay is much larger than that of low frequency bands, which leads to a much lower delay spread. In the angular domain, although the scattering paths appear everywhere, dominant paths are usually sparsely distributed, so the angular spreads are comparable to those of low frequency bands.
Table III

COMPARISON OF THE LARGE-SCALE CHANNEL CHARACTERISTICS.

\begin{tabular}{l|cccc}
\hline & Measured & SC+DC & SC & WINNER \\
\hline Channel power $[\mathrm{dBm}]$ & -45.78 & -45.83 & -50.32 & $/$ \\
Delay spread $\left[\log _{10}(s)\right]$ & -8.31 & -8.30 & -8.44 & -7.6 \\
Azim. spread $\left[\log _{10}\left({ }^{\circ}\right)\right]$ & 1.35 & 1.37 & 1.11 & 1.73 \\
Elev. spread $\left[\log _{10}\left({ }^{\circ}\right)\right]$ & 1.57 & 1.64 & 1.35 & $/$ \\
\hline
\end{tabular}

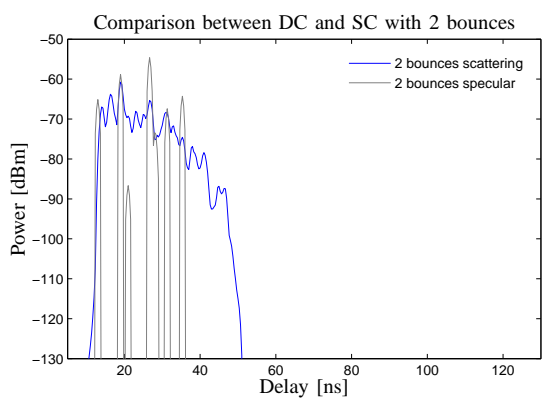

(a)

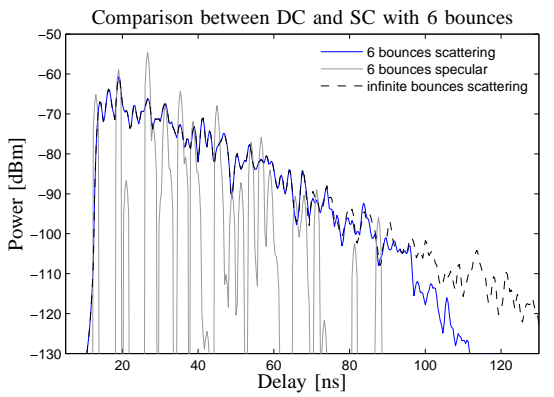

(b)

Figure 15. Comparison of the power delay profiles between the diffuse and specular components with (a) two bounces and (b) six bounces.

\section{COMPARISON BETWEEN DC AND SC WITH REVERBERATION}

The previous results showed that the DMC (background in the PAS and tail in the PDP) can be well grasped by using the graph modelling to simulate the DC. Intuitively speaking, the DMC should contain not only the DC but also the reverberation part of the SC. However, according to the previous PDP comparisons, the latter seems to have little influence on the diffuse tail. Therefore, in order to investigate the different behaviors of DC and SC in terms of reverberation effect, we conduct an additional simulation in an empty room environment at $60 \mathrm{GHz}$. The size of the room is the same as the indoor office scenario, but the furniture is removed except the absorber, in order to reduce simulation complexity while maintaining the NLoS condition.

Figure 15 shows the comparison between the graph modelling-simulated DC and the RT-simulated SC, considering 2 bounces and 6 bounces respectively. The simulated DC with infinite bounces is also depicted as a reference. The scattering coefficient $S$ is set to be 0.6 in graph modelling and only the reflection mechanism is activated in RT tool. It can be observed that 1) the SC appears discrete with many null intervals in the delay domain, while the DC is continuous: this is because of 
the distributed nature of the DC as opposed to the concentrated nature of the $\mathrm{SC} ; 2$ ) there are some differences on the power level in the initial part of the PDPs, especially at the delay around $30 \mathrm{~ns}$. This is due to the fact that the propagation attenuation of the diffuse scattering versus distance is greater than that of the reflection; 3 ) as the number of bounces increases from 2 to 6 , the PDP tails are prolonged, as they should, with the same discrete vs. continuous difference between the SC and the DC; 4) there is less power difference in the tails due to the reason that, although the power of individual DC paths is much lower than that of specular paths, the time-density of scattering paths is much larger, especially after several bounces of reverberation. For a finite-bandwidth propagation channel the path components within each delay bin overlap, so that the total power of DC within each delay bin is comparable to the SC power. Therefore, it seems reasonable to approximate the DMC reverberation tail by the DC-only reverberation tail. These results confirm the validity of our approach which describes the whole propagation channel as the combination of the SC with a limited number of bounces and of the DC with an infinite number of bounces.

\section{CONCLUSION}

In this contribution, we propose a semi-deterministic channel modelling approach based on graph theory and ray-tracing. The "Effective Roughness" model is applied into propagation graph modelling to simulate the diffuse scattering component, while the specular components are simulated through raytracing. The implementations of the proposed method and the derivations of the propagation coefficients are illustrated in detail. Two reference scenarios on different frequencies are adopted for performance evaluations. The simulated power delay profiles and power angular spectra are compared with those obtained through the original graph modelling method, ray tracing and measurement data. The results show that our proposed approach can realistically simulate the propagation channel in both the delay and angular domains, including the reverberant component, in a reasonable computation time.

\section{ACKNOWLEDGMENT}

The authors would like to thank Prof. Claude Ostges, Dr. Francesco Mani, UCL, and Prof. Reiner Thomae, Diego Dupleich, Technical University of Ilmenau, for providing us with the measurement data and for their assistance in the data post-processing.

\section{REFERENCES}

[1] M. Landmann, K. Sivasondhivat, J.-I. Takada, I. Ida, and R. Thomä, "Polarization behavior of discrete multipath and diffuse scattering in urban environments at $4.5 \mathrm{GHz}$," EURASIP Journal on Wireless Communications and Networking, vol. 2007, no. 1, pp. 60-60, 2007.

[2] V. Degli-Esposti, D. Guiducci, A. de'Marsi, P. Azzi, and F. Fuschini, "An advanced field prediction model including diffuse scattering," IEEE Transactions on Antennas and Propagation, vol. 52, no. 7, pp. 17171728, 2004.

[3] J. Poutanen, J. Salmi, K. Haneda, V. Kolmonen, F. Tufvesson, and P. Vainikainen, "Propagation characteristics of dense multipath components," Antennas and Wireless Propagation Letters, IEEE, vol. 9, pp. 791-794, 2010.
[4] J. Järveläinen and K. Haneda, "Sixty gigahertz indoor radio wave propagation prediction method based on full scattering model," Radio Science, vol. 49, no. 4, pp. 293-305, 2014.

[5] C. Jansen, S. Priebe, C. Moller, M. Jacob, H. Dierke, M. Koch, and T. Kurner, "Diffuse scattering from rough surfaces in $\mathrm{THz}$ communication channels," IEEE Transactions on Terahertz Science and Technology, vol. 1, no. 2, pp. 462-472, Nov 2011.

[6] V. Degli-Esposti, F. Fuschini, E. M. Vitucci, M. Barbiroli, M. Zoli, L. Tian, X. Yin, D. Dupleich, R. Muller, C. Schneider, and R. Thoma, "Ray-tracing-based mm-wave beamforming assessment," IEEE Access, vol. 2, pp. 1314-1325, 2014.

[7] A. Richter, "Estimation of radio channel parameters: Models and algorithms." ISLE, 2005.

[8] V. Degli-Esposti, "A diffuse scattering model for urban propagation prediction," IEEE Transactions on Antennas and Propagation, vol. 49, no. 7, pp. 1111-1113, 2001.

[9] D. Didascalou, M. Dottling, N. Geng, and W. Wiesbeck, "An approach to include stochastic rough surface scattering into deterministic rayoptical wave propagation modeling," IEEE Transactions on Antennas and Propagation, vol. 51, no. 7, pp. 1508-1515, 2003.

[10] D. Ullmo and H. U. Baranger, "Wireless propagation in buildings: A statistical scattering approach," IEEE Transactions on Vehicular Technology, vol. 48, no. 3, pp. 947-955, 1999.

[11] T. Pedersen and B. Fleury, "Radio channel modelling using stochastic propagation graphs," in IEEE International Conference on Communications, 2007. ICC '07, june 2007, pp. 2733 -2738.

[12] T. Pedersen, G. Steinbock, and B. Fleury, "Modeling of reverberant radio channels using propagation graphs," IEEE Transactions on Antennas and Propagation, vol. 60, no. 12, pp. 5978-5988, 2012.

[13] L. Tian, X. Yin, Q. Zuo, J. Zhou, Z. Zhong, and S. X. Lu, "Channel modeling based on random propagation graphs for high speed railway scenarios," in IEEE 23rd International Symposium on Personal Indoor and Mobile Radio Communications (PIMRC), 2012, pp. 1746-1750.

[14] L. Tian, X. Yin, X. Zhou, and Q. Zuo, "Spatial cross-correlation modeling for propagation channels in indoor distributed antenna systems," EURASIP Journal on Wireless Communications and Networking, vol. 2013, no. 183, pp. 1-11, 2013.

[15] P. Kyosti, J. Meinila, L. Hentila, X. Zhao, T. Jamsa, C. Schneider, M. Narandzic, M. Milojevic, A. Hong, J. Ylitalo et al., "Winner II channel models," European Commission, IST-WINNER D, vol. 1.

[16] J. Jarvelainen, K. Haneda, M. Kyro, V.-M. Kolmonen, J. Takada, and H. Hagiwara, "60 GHz radio wave propagation prediction in a hospital environment using an accurate room structural model," in Antennas and Propagation Conference (LAPC), 2012 Loughborough, 2012, pp. 1-4.

[17] C. Jansen, S. Priebe, C. Möller, M. Jacob, H. Dierke, M. Koch, and T. Kürner, "Diffuse scattering from rough surfaces in $\mathrm{THz}$ communication channels," IEEE Transactions on Terahertz Science and Technology, vol. 1, no. 2, pp. 462-472, 2011.

[18] F. Fuschini, E. M. Vitucci, M. Barbiroli, G. Falciasecca, and V. DegliEsposti, "Ray tracing propagation modeling for future small-cell and indoor applications: a review of current techniques," Radio Science, vol. 50, no. 6, pp. 469-485, June 2015.

[19] L. Tian, V. Degli-Esposti, E. M. Vitucci, X. Yin, F. Mani, and S. X. Lu, "Semi-deterministic modeling of diffuse scattering component based on propagation graph theory," in IEEE 25rd International Symposium on Personal Indoor and Mobile Radio Communications, 2014, pp. 1-5.

[20] V. Degli-Esposti, F. Fuschini, E. M. Vitucci, and G. Falciasecca, "Measurement and modelling of scattering from buildings," IEEE Transactions on Antennas and Propagation, vol. 55, no. 1, pp. 143-153, 2007.

[21] V. Degli-Esposti, F. Fuschini, and E. M. Vitucci, "A fast model for distributed scattering from buildings," in 3rd European Conference on Antennas and Propagation (EuCAP). IEEE, 2009, pp. 1932-1936.

[22] H. Friis, "A note on a simple transmission formula," proc. IRE, vol. 34, no. 5 , pp. 254-256, 1946.

[23] U. Bakshi and A. Bakshi, Antenna And Wave Propagation. Technical Publications, 2009.

[24] E. M. Vitucci, F. Mani, V. Degli-Esposti, and C. Oestges, "Polarimetric properties of diffuse scattering from building walls: Experimental parameterization of a ray-tracing model," IEEE Transactions on Antennas and Propagation, vol. 60, no. 6, pp. 2961-2969, 2012.

[25] C. A. Balanis, Advanced engineering electromagnetics. Wiley New York, 1989, vol. 205.

[26] D. Dupleich, F. Fuschini, R. Mueller, E. M. Vitucci, C. Schneider, V. Degli-Esposti, and R. Thomä, "Directional characterization of the $60 \mathrm{GHz}$ indoor-office channel," in 31th URSI General Assembly and Scientific Symposium, Beijing, China, 2014, pp. 1-5. 


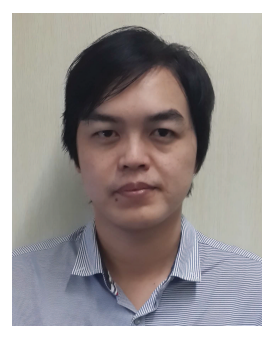

Li Tian received the bachelor degree in communication engineering and the Ph.D degree in Control Science and Control Engineering from Tongji University, Shanghai, China, in July 2009 and January 2015, respectively. From 2013 to 2014, he was a visiting Ph.D student at the Department of Electronics and Information Systems (DEIS), University of Bologna, working with Prof. Vittorio Degli-Esposti.

$\mathrm{He}$ participated in the $5 \mathrm{G}$ project sponsored by National Natural Science Foundation of China. He is now a Senior Engineer at the Department of Algorithms, ZTE Corporation. His current research interests are in the field of $5 \mathrm{G}$ channel modeling and new air-interface.

Dr. Tian serves as reviewer for a number of international journals including IEEE Transactions on Antennas and Propagation, IEEE Transactions on Vehicular Technology, IEEE Access, IEEE Antennas and Wireless Propagation Letters, and International Journal of Antennas and Propagation.

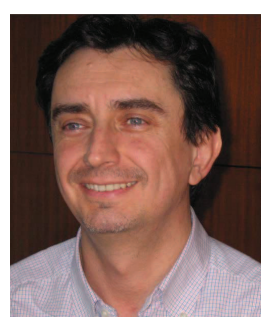

Vittorio Degli-Esposti (M'94) received the 'Laurea' degree (with Honours) and the Ph.D. degree in Electronic Engineering from the University of Bologna, Italy, in 1989 and in 1994, respectively. From 1989 to 1990 he was with Siemens Telecomunicazioni, Milan, in the microwave communications group. Since November 1994 he has been with the Department of Electrical Engineering (DEI) of the University of Bologna, where he is now Associate Professor and teaches courses on Electromagnetics, Radio Propagation and wireless systems.

Since 2015 he is on leave from the University position and joined Polaris Wireless Inc., Mountain View, California, USA in the role of Director of Research. He was a visiting researcher in 1998 at the Polytechnic University, Brooklyn, New York (now NYU Polytechnic Institute) where he worked with Professor H.L. Bertoni. In 2006 and 2013 he held Visiting Professor Positions at Helsinki University of Technology (now Aalto University), and at Tongji University, Shanghai, respectively, to teach courses on "Deterministic propagation modeling and ray tracing". Prof. Degli-Esposti still has ongoing research collaborations with the above-mentioned Institutions and several other Universities and Companies worldwide.

Vittorio Degli-Esposti participated in the European Cooperation Actions COST COST 231, 259, 273, 2100 and IC1004, in the European Networks of Excellence NEWCOM and NEWCOM++ in the European Project ALPHA and in several other national and international projects.

$\mathrm{He}$ is author or co-author of about 100 peer-reviewed technical papers in the fields of applied electromagnetics, radio propagation and wireless systems. $\mathrm{He}$ is Associate Editor of the scientific Journal "IEEE Access" and serves as reviewer for a number of IEEE Transactions.

He chaired, organized sessions and served in the Technical Program Committees at several International Conferences, including all EuCAP editions. $\mathrm{He}$ has been appointed Vice-Chair of EuCAP2010 and EuCAP2011. He has been an elected member of the Radio Propagation Board of the European Association on Antennas and Propagation (EuRAAP) from 2013 to 2015.

$\mathrm{He}$ is co-organizer and lecturer of the European School of Antennas Course "Short range radio propagation: theory, models and future applications". In 2013 he has been elected Chair of the Cesena-Forli Unit of the InterDepartment Center for Industrial Research on ICT (CIRI-ICT) of the University of Bologna.

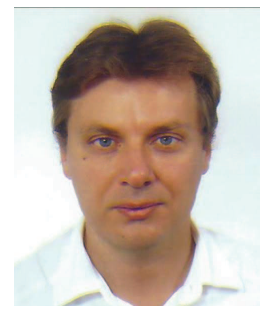

Enrico M. Vitucci (S04CM08) received the M.Sc. degree in Telecommunication Engineering and the Ph.D. degree in Electrical Engineering and Computer Science from the University of Bologna, Italy, in 2003 and 2007, respectively. Currently, he is a Postdoctoral Fellow at the Center for Industrial Research on ICT (CIRI ICT), University of Bologna. In 2007, he was a visiting researcher at the Helsinki University of Technology C TKK, now Aalto University, Finland. From October 2015 to March 2016, he was a visiting researcher at Polaris Wireless, Inc., Silicon Valley, California (USA). His research interests are in mobile radio propagation, ray tracing models, MIMO channel modelling, and energy efficiency in urban areas. He participated in the European Cooperation Actions COST 273, COST 2100, COST IC1004, in the European Networks of Excellence FP6-NEWCOM and FP7-NEWCOM++, and in the EU Integrated Project FP7-ICT-ALPHA. He authored or co-authored about 50 technical papers on international journals or conferences. He also serves as reviewer for a number of international journals and conferences, including several IEEE TRANSACTIONS.

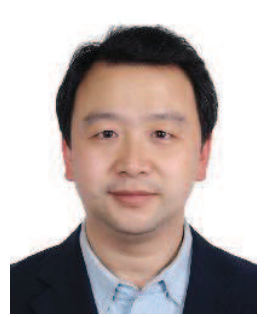

Xuefeng Yin received his Bachelor degree in optoelectronics engineering from Huazhong University of Science and Technology, Wuhan, China, in 1995, and his MS in digital communications and $\mathrm{PhD}$ in wireless communications from Aalborg University, Denmark, in 2002 and 2006, respectively. From 2006 to 2008 , he worked as assistant professor in Aalborg University. In 2008, he joined Tongji University as associate professor in the college of electronics and information engineering, Shanghai, China. His research interests include high-resolution parameter estimation for propagation channels, channel measurement, channel characterization and stochastic modelling, and radar signal processing. 